\title{
INADEQUAÇÃO DE TROMBOPROFILAXIA VENOSA EM PACIENTES CLÍNICOS HOSPITALIZADOS
}

\author{
Ana Thereza C. Rocha*, Priscila Braga, Guilherme Ritt e Antônio Alberto Lopes \\ Trabalho realizado no Departamento de Medicina da Faculdade de Medicina da Bahia da Universidade Federal da Bahia (UFBA)
}

\section{*Correspondência:}

Rua Alberto Valença, 148

Ed. Mansão do Lena

apto. 203

41810-825 - Salvador - BA

anatrocha@alumni.duke.edu

\section{RESUMO}

O risco de tromboembolismo venoso (TEV) é alto em pacientes internados, mas pode ser reduzido com a utilizaçãa adequada de profilaxia.

OBjetivo. Avaliar a utilização e adequação de profilaxia para TEV em pacientes clínicos internados.

Métodos. Estudo de coorte transversal em pacientes internados por doenças clínicas em quatro hospitais de Salvador.

Resultados. Foram avaliados 226 pacientes: 15,5\% em UTIs clínicas, $79 \%$ tinham idade $\geq 40$ anos e $48 \%$ eram homens. A maioria (97\%) apresentava pelo menos um fator de risco (FR) para TEV: mobilidade reduzida em $79 \%$ e diagnóstico principal como FR em 62\%. Dos 208 candidatos a profilaxia, 54\% receberam alguma forma: heparina não fracionada (HNF) em 44\%, heparina de baixo peso molecular (HBPM) em $56 \%$ e métodos mecânicos em dois pacientes. A taxa de utilização foi semelhante entre hospitais privados e públicos (51\% versus $49 \%)$, mas HBPM predominou em privados $(97 \%)$ e sem residência médica e HNF em públicos (86\%). HBPM foi usada mais freqüentemente que HNF em pacientes $\geq 40$ anos, em brancos que em negros ou mulatos, e menos freqüentemente em pacientes com contra-indicações para heparina. Dos 1 I 2 pacientes com profilaxia, $63 \%$ receberam dosagem adequada: HBPM em 95,2\% e HNF em 20,4\%. Profilaxia para TEV foi adequada em apenas 33,6\% (70/208) dos pacientes.

ConcLusÄo. FR para TEV são freqüentes em pacientes clínicos. Existe ampla variabilidade da profilaxia prescrita em hospitais públicos e privados. HBPM é utilizada mais adequadamente que HNF, entretanto, apenas a minoria dos pacientes clínicos hospitalizados e candidatos a profilaxia recebem dosagem adequada.

Unitermos: Tromboembolismo. Fatores de risco. Prevenção e controle. Medicina Interna. Heparina. Diretrizes para a prática clínica.

\section{INTRODUÇÃO}

O risco de tromboembolismo venoso (TEV) é alto tanto em pacientes cirúrgicos quanto naqueles internados para tratamento de doenças clínicas. Os resultados de diversos estudos controlados e randomizados, no entanto, têm servido para mostrar intervenções capazes de reduzir de forma marcante o risco de TEV nestes pacientes ${ }^{1,2,3,4,5,6}$. Diretrizes sobre profilaxia de TEV têm sido elaboradas tomando por base os resultados destes ensaios clínicos ${ }^{7,4.8}$. Apesar disso, vários estudos mostram grande variabilidade na utilização de profilaxia de TEV, sendo freqüente a inobservância às orientações dos consensos internacionais $9,10,11$. Em um estudo realizado entre 1996 e 1997 em um grande hospital universitário de Montreal, Arnold et al. ${ }^{12}$ demonstraram que cerca de 17,4\% dos casos de TEV eram potencialmente preveníveis. Motivos freqüentemente observados para falha na prevenção foram: omissão de profilaxia (48\%), duração inadequada (23\%) e escolha incorreta do método de profilaxia (2।\%).

No Brasil, existe uma carência de dados que permitam estimativas acuradas do risco de TEV em pacientes clínicos e da adequação dos métodos de profilaxia atualmente empregados. Em estudo recente avaliando conjuntamente a adequação de profilaxia de TEV em pacien- tes clínicos e cirúrgicos, em quatro hospitais de São Paulo, de acordo com as recomendações do American College of Chest Physicians de $200 \mathrm{I}^{4}$, os autores mostraram que $37 \%$ dos pacientes cirúrgicos e com alto risco de TEV receberam profilaxia inadequada ${ }^{13}$. Além disso, a adequação da profilaxia foi maior para pacientes cirúrgicos que clínicos ( $50 \%$ versus $36 \%$, respectivamente, $p<0,05$ ). Visando preencher esta lacuna, a Associação Médica Brasileira tem estimulado o desenvolvimento de pesquisas no país e a elaboração de diretrizes baseadas em evidência com o intuito de melhorar a compreensão do risco de TEV em pacientes clínicos e adequar a utilização de profilaxia. O presente estudo faz parte desta iniciativa e visa avaliar a frequêencia da utilização de profilaxia para TEV em pacientes clínicos internados em hospitais antes da divulgação da Diretriz Brasileira para Profilaxia de TEV em Pacientes Clínicos. O presente estudo tem também o objetivo de avaliar os fatores associados à utilização e às variações entre os métodos de profilaxia empregados.

\section{Métodos \\ Desenho}

Estudo observacional, de coorte transversal, em que os dados dos pacientes clínicos internados foram coletados em um único dia em 
março de 2005, antes da divulgação das recomendações da Diretriz Brasileira para Profilaxia de TEV em Pacientes Clínicos. Um investigador em cada hospital participante teve acesso ao registro geral de pacientes internados e fez a triagem de todos aqueles $\geq 18$ anos que foram internados para tratamento clínico. Pacientes internados no dia do estudo e com estadia esperada $<48$ horas e aqueles submetidos a cirurgia na semana antecedente foram excluídos. Com exceção dos representantes médicos de cada hospital, os outros médicos locais estavam desinformados sobre a ocorrência do estudo naquela data, para evitar modificação na prescrição de profilaxia. Os hospitais escoIhidos em Salvador, Bahia, não tinham programas formais de incentivo à profilaxia contra TEV; foram eles: Hospital Português, Hospital Universitário Professor Edgard Santos, Hospital Geral Roberto Santos e Hospital Aliança.

Foram utilizados questionários padronizados para coleta de dados a partir dos prontuários e entrevistas com os pacientes. Foram coletados dados sobre características de cada hospital (número de leitos, tipo [ex. público ou particular] e presença de programa de residência médica ou não), características demográficas dos pacientes, fatores de risco (FR) para TEV (história pessoal ou familiar de $T E V$, neoplasia, quimioterapia, hormonoterapia, gravidez, puerperium, trombofilia hereditária ou adquirida, terapia de reposição hormonal, cateteres venosos centrais e obesidade) e comorbidades (insuficiência cardíaca congestiva [ICC], infarto agudo do miocárdio [IAM], insuficiência vascular periférica, varizes, paresia/paralisia, acidente vascular cerebral isquêmico $[\mathrm{AVCl}]$, acidente vascular cerebral hemorrágico $[\mathrm{AVCH}]$, insuficiência respiratória com e sem ventilação mecânica, doença pulmonar obstrutiva crônica [DPOC], pneumonia, doenças inflamatórias intestinais, doenças reumáticas ativas, infecções [exceto pneumonia] e síndrome nefrótica).

Foram coletados também dados sobre o uso de profilaxia contra TEV da seguinte maneira: I) HNF, HBPM ou warfarina I mg/dia para profilaxia de CVC; 2) para HBPM, dose diária (ex: $20 \mathrm{mg}, 40 \mathrm{mg}$ de enoxaparina ou doses equivalentes de outras HBPM); 3) contraindicações para heparina (cirurgia ocular ou cerebral a menos de duas semanas, insuficiência renal crônica, alergia a heparina, sangramento ativo, definido como sangramento macroscópico de qualquer local, excluindo locais de punção venosa; ou coagulopatia, definida como coagulação intravascular disseminada, plaquetas $<50.000 / \mathrm{mm}^{3}$, tempo de tromboplastina parcial ativada (PTT) $>60$ segundos ou international normalized ratio (INR) $>2,0$ sem uso de warfarina); 4) prescriçãa de métodos físicos de profilaxia (meias elásticas de compressão gradual [MECG] ou compressão pneumática intermitente $[\mathrm{CPI}]$ ); 5) uso terapêutico de anticoagulantes (HNF ou HBPM em doses destinadas à anticoagulaçãa sistêmica).

Foram calculados os intervalos de tempo entre a admissão do paciente e início da profilaxia, a duração relativa da profilaxia (intervalo de tempo entre o início da profilaxia e a data do estudo) e a adequação da profilaxia (dosagem adequada de HNF ou HBPM para pacientes clínicos hospitalizados, considerados candidatos a profilaxia pela presença de pelo menos um fator de risco para TEV), de acordo com as recomendações da Diretriz Brasileira para Profilaxia de TEV em Pacientes Clínicos (publicação em andamento no Projeto Diretrizes AMB-CFM, www.projetodiretrizes.org.br).
Os comitês institucionais locais de ética em pesquisa concederam aprovação para realização do estudo e um termo de consentimento livre e esclarecido foi obtido de todos os pacientes.

\section{Análise estatística}

Os dados são apresentados como proporções, médias e desvios padrão ou medianas e distância interquartílica (IQR ou interquartile range), quando apropriado. As variáveis quantitativas foram comparadas usando o teste t de Student, quando a distribuição era normal ou teste da mediana para dados não-paramétricos. Variáveis categóricas foram comparadas com teste Qui-quadrado ou teste Exato de Fisher, quando apropriado. A idade dos pacientes foi tratada como variável contínua e como categórica, utilizando 40 anos como ponto de corte. Aqueles pacientes recebendo anticoagulação sistêmica e aqueles com diagnóstico de TEV à admissão foram excluídos das análises. Os diagnósticos de admissão foram categorizados como FR para TEV ou não. Os FR para TEV, de acordo com a história clínica, foram avaliados pelo número total e categoricamente entre nenhum, I a 3 ou $\geq 4$. A variável contra-indicação para profilaxia farmacológica foi criada de acordo com a presença de nenhum ou $\geq$ I dos seguintes: diagnóstico principal com risco de sangramento (ex: sangramento gastrointestinal), FR para sangramento pela história clínica e presença de contra-indicações para heparina citadas acima. Foram feitas análises comparativas para estimar: I) freqüência do uso de profilaxia em geral e de acordo com tipo de hospital (público ou particular, presença de residência médica ou não); 2) fatores associados ao uso de qualquer profilaxia versus nenhuma; 3) fatores associados ao uso profilaxia com HNF versus HBPM; 4) adequação de profilaxia baseada nas recomendações da Diretriz Brasileira para Profilaxia de TEV em Pacientes Clínicos, definida como uso de profilaxia em paciente hospitalizado com pelo menos um FR para TEV, além de mobilidade reduzida, sem contra-indicações para heparina e com doses profiláticas altas do método escolhido. Foram realizados testes de Qui-quadrado ou Fisher para estimar fatores potenciais associados ao uso de profilaxia. Todas as análises, inclusive de regressão logística multivariadas para identificar fatores independentemente associados com a utilização de profilaxia, foram feitas com o software SPSS 9.0 for Windows. As razões de chances (odds ratios ou OR) são apresentadas, juntamente com os respectivos intervalos de confiança. Valores de $p<0,05$ estabeleceram o nível de significância estatística.

\section{Resultados}

Quatro hospitais participaram do estudo: dois privados e com residência médica e dois públicos e sem residência médica. O número médio de leitos clínicos ocupados foi de $72 \pm 14$. Foram recrutados 226 pacientes clínicos hospitalizados e cada hospital contribuiu com 23\% a 26,5\% dos pacientes. Cerca de 15,5\% dos pacientes pertenciam a unidades de terapia intensiva (UTI) clínicas ou mistas.

A média de idade dos pacientes foi de 58,8 \pm 19,2 anos (79,2\% com idade $\geq 40$ anos), sendo $47,8 \%$ do sexo masculino. Dos 226 pacientes, 78,8\% apresentavam mobilidade reduzida. Os dados demográficos e outras características dos pacientes recrutados são 


\begin{tabular}{|c|c|}
\hline \multicolumn{2}{|c|}{ Tabela I - Características gerais dos pacientes clínicos hospitalizados } \\
\hline Variáveis & $\begin{array}{l}\text { Pacientes recrutados } \\
(\mathrm{N}=226)\end{array}$ \\
\hline Idade, média \pm DP & $58,1 \pm 19,2$ \\
\hline$\geq 40$ anos & $79,2(179)$ \\
\hline Gênero masculino & $47,8(108)$ \\
\hline \multicolumn{2}{|l|}{ Raça } \\
\hline Branca & $51,3(116)$ \\
\hline Negra & $20,8(47)$ \\
\hline Mulata & $27,9(63)$ \\
\hline Peso, média \pm DP & $61,7 \pm|5|$, \\
\hline Altura, média \pm DP & $1,63 \pm 0,49$ \\
\hline IMC, média \pm DP & $23,3 \pm 5,2$ \\
\hline \multicolumn{2}{|l|}{ Tipo de hospital onde os pacientes foram internados } \\
\hline Público & $50,4(|| 4)$ \\
\hline Privado & $49,6(112)$ \\
\hline Internado em hospital com programa de residência médica & $50,4(|| 4)$ \\
\hline \multicolumn{2}{|l|}{ Diagnóstico principal de admissão } \\
\hline Insuficiência cardíaca congestiva & $5,3(\mid 2)$ \\
\hline Pneumonia & $7,5(17)$ \\
\hline Infarto agudo do miocárdio & $3,5(8)$ \\
\hline Cardíaco outro & $4,9(||)$ \\
\hline Respiratório outro & $4,9(||)$ \\
\hline Acidente vascular cerebral isquêmico & $7,1(16)$ \\
\hline Acidente vascularcerebral hemorrágico & $3,5(8)$ \\
\hline Neurológicos outros & $4,0(9)$ \\
\hline Doença inflamatória intestinal & $2,7(6)$ \\
\hline Gastrointestinal outro & $8,4(19)$ \\
\hline Renal & $13,7(3 \mid)$ \\
\hline Infecção localizada & $3,5(8)$ \\
\hline Infecção sistêmica & $3,1(7)$ \\
\hline Câncerhematológico & $4,9(||)$ \\
\hline Câncer de órgão sólido & $6,2(14)$ \\
\hline Doença reumática ativa & $2,7(6)$ \\
\hline Doença com risco de hemorragia & $2,2(5)$ \\
\hline Outros & $8,8(20)$ \\
\hline TEV & $3,1(7)$ \\
\hline Diagnóstico principal é FR para TEV & $66,8(|5|)$ \\
\hline FR para TEV $(\geq \mathrm{I})$ excluindo mobilidade reduzida & $87,6(198)$ \\
\hline Mobilidade reduzida & $78.8(178)$ \\
\hline Contra-indicação para heparinas & $36,3(82)$ \\
\hline
\end{tabular}

DP indica desvio padrão; FR, fatores de risco; TEV, tromboembolismo pulmonar

mostrados na Tabela I. Dezoito pacientes foram excluídos das análises: 7 porque tiveram o diagnóstico de TEV à admissão e I I por receberem anticoagulação sistêmica em função do diagnóstico de admissão. Estes pacientes, portanto, não foram candidatos a outros métodos de profilaxia para TEV.

Dos 208 pacientes candidatos à profilaxia, 53,8\% receberam alguma forma de profilaxia de TEV. A mediana do intervalo entre a admissão e o início da profilaxia foi de I dia (IQR 0 a 4) e a mediana do tempo de uso foi de 12 dias (IQR 5 a 28). A Tabela 2 mostra variáveis que potencialmente influenciaram o uso de profilaxia. Entre os fatores de risco para tromboembolismo venoso, houve freqüência significativamente maior de câncer de órgão sólido ( $14,7 \%$ versus $5,1 \%, p<$ $0,02), \operatorname{AVCH}(22,0 \%$ versus $8,1 \%, p<0,005)$, doença respiratória grave $(\mid 4,7 \%$ versus $5, \mid \%, p<0,02)$, paresia/paralisia de membros inferiores $(38,5 \%$ versus $22,2 \%, p<0,01)$ e admissão em UTI $(2|| \$,$% versus 9,|\%, p<0,0|$ ) entre os pacientes que receberam e os que não receberam profilaxia.

Quando comparados com pacientes que não receberam profilaxia, aqueles que receberam eram significativamente mais velhos $(62 \pm 18$ versus $53 \pm 19$ anos, $p=0,001$ ), apresentavam mais freqüentemente o diagnóstico principal como FR para TEV (70\% versus $48 \%, p=$ 0,001 ) e mobilidade reduzida ( $84 \%$ versus $73 \%, p=0,05$ ), tinham mais freqüentemente $\geq 4 \mathrm{FR}$ para TEV, excluindo mobilidade reduzida $(41 \%$ versus $19 \%, p=0,002)$ e menos contra-indicações para heparinas (29\% versus 36\%, $p=0,01$ ).

Análise de regressão logística com eliminação retrógrada de variáveis mostrou que, dos fatores associados à utilização de profilaxia, apenas idade $\geq 40$ anos (OR 2,07, intervalo de confiança [IC] de $95 \%$ I,03 a 4, I 6, $p=0,04$ ) e o número de fatores de risco de TEV (OR de 2,92, IC de $95 \% 1,10$ a 7,73 para FR $\geq 4$ versus 0 e OR de I, $03, I C$ de $95 \% 0,43$ a 2,49 para $F R \geq 4$ versus $\mid-3, p=0,006$ ) foram independentemente associados à maior utilização de profilaxia. Enquanto a presença de contra-indicações absolutas ou relativas para heparinas foi associada à menor utilização de profilaxia (OR 0,47, IC de $95 \%$ 0,27 a 0,84, $p=0,0$ I). O efeito do diagnóstico principal como FR para TEV e o da mobilidade reduzida foram eliminados durante a avaliação multifatorial.

Entre os pacientes que receberam profilaxia, HNF foi usada em 43,8\% e HBPM em 56,3\%. MECG foram usadas concomitantemente com métodos farmacológicos em apenas dois pacientes e nenhum paciente recebeu $\mathrm{CPI}$. O intervalo de tempo entre a admissão ao hospital e o início da profilaxia não diferiu entre aqueles que receberam HBPM ou HNF, mediana de $0(0$ a 2$)$ dias versus $2(0$ a 8$)$ dias, $p=0,18$. A duração da profilaxia foi inferior entre aqueles que receberam HBPM e os que receberam HNF, mediana de 9 ( 3 a 27) dias versus 16 ( 9 a 43) dias, $p=0,05$. A duração da profilaxia também diferiu entre hospitais públicos e privados, mediana de 19 (9 a 43) versus 8 (3 a 19,5), respectivamente, $p=0,01$. Fatores associados ao tipo de profilaxia de tromboembolismo venoso utilizada são mostrados na Tabela 3.

A utilização de profilaxia para TVP foi similar entre pacientes de hospitais privados e públicos (51\% versus $49 \%, p=0,39)$, sendo observado, no entanto, diferença estatisticamente significante no tipo de heparina utilizada. Os percentuais de pacientes utilizando HBPM em hospitais públicos e privados foram 12,7\% e 97,6\%, respectivamente, $p<0,001$. A idade dos pacientes também influenciou o uso de profilaxia: HBPM foi prescrita para uma maior porcentagem de pacientes $\geq 40$ anos que HNF ( $92 \%$ versus $75 \%, p=0,02$ ). $O$ gênero dos pacientes não foi associado com o tipo de prescrição, mas a raça dos pacientes sim: HBPM foi usada em mais pacientes da raça branca que da raça negra ou mulata (69,8\% versus $30,1 \%)$. Entretanto, HNF foi usada em menos pacientes da raça branca que da raça negra ou mulata ( $24,5 \%$ versus $75,5 \%), p<0,000$ I. Notamos que significativamente mais pacientes em hospitais privados tinham idade $\geq 40$ anos que em hospitais públicos ( $92 \%$ versus $67 \%, p<0,000$ I). Do mesmo modo, mais pacientes da raça branca foram hospitalizados em hospitais privados que públicos (79\% versus 25\%, p < 0,000 I), onde a prescrição de HBPM foi bastante superior. 


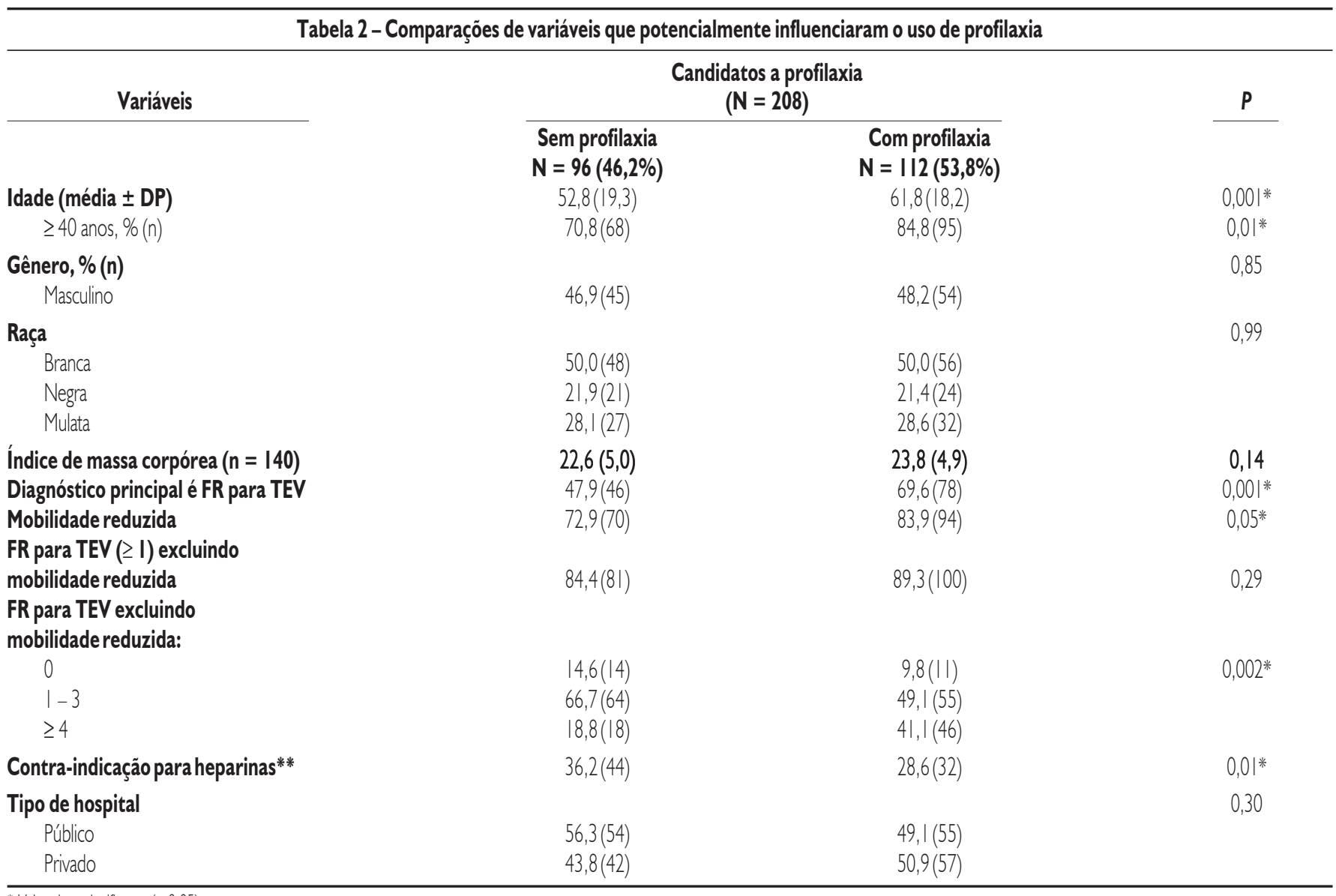

* Valor de $p$ significante $(<0,05)$

***ontra-indicaçōes para heparinas incluindo sangramento ativo, coagulopatia, insuficiência renal crônica, alergia, trombocitopenia induzida por heparina (HIT), cirurgia ocular ou craniana

Pacientes com FR para sangramento e/ou contra-indicações para profilaxia farmacológica receberam HNF mais freqüentemente que HBPM ( $47 \%$ versus I $4 \%, p<0,000$ I). Mais pacientes com diagnósticos de admissão que são FR para TEV receberam HBPM que HNF (78\% versus $59 \%, p=0,03)$. O número de FR para TEV alterou a prescrição de HNF ou HBPM (61,2\% versus $39,7 \%$ se $\mid-3$ FR e $28,6 \%$ versus $50,8 \%$ se $\geq 4 F R$, respectivamente, $p=0,05$ ).

Quanto à adequação da profilaxia, a maioria dos pacientes que receberam HBPM teve dosagem adequada: 79,4\% receberam $40 \mathrm{mg} /$ dia, $4,8 \%, 20 \mathrm{mg} /$ dia e $15,9 \%$ outras doses. Entretanto, apenas $20,4 \%$ dos pacientes receberam HNF 5.000 UI de 8-8h, enquanto que 79,6\% receberam $5.000 \mathrm{UI}$ de I2-12h. Considerando-se que apenas a dosagem de HNF mais alta (5.000 UI de 8-8h) tem sido comprovadamente equivalente às HBPM em doses profiláticas altas, podemos dizer que, dos 1 I 2 pacientes que receberam profilaxia, apenas 63\% (70/I I2) receberam dosagem adequada. Destes, uma proporção significativamente superior recebeu dosagem adequada de HBPM do que HNF (86\% versus I $4 \%, p<0,000 I$ ). Assim, apenas 33,6\% (70/208) dos pacientes clínicos hospitalizados e candidatos a profilaxia receberam dosagem adequada.

\section{Discussão}

Existe uma carência de dados sobre a utilização de profilaxia de TEV em pacientes clínicos em nível nacional. O presente estudo de coorte transversal realizado em hospitais de Salvador deve ser visto como uma iniciativa para a avaliação da situação atual da profilaxia de TEV em nosso meio. Este tipo de levantamento deveria ser realizado em outras cidades brasileiras para alertar a comunidade médica sobre a freqüente inadequação do uso de profilaxia para TEV em hospitais tanto públicos como privados. Neste estudo, mostrou-se que profilaxia em pacientes clínicos é subutilizada, e, quando empregada, freqüentemente não adere às recomendações de consensos e diretrizes. Razões freqüentes para isso foram falta de uma avaliação sistemática de fatores de risco para TEV e de contra-indicações para heparinas, e também o uso de doses de heparinas não eficazes para estes pacientes. É importante notar que, apesar de não ter havido diferença significativa entre a utilização de profilaxia, em geral, o percentual de pacientes utilizando HBPM foi significantemente maior em hospitais privados que públicos. Embora ambos os tipos de heparina estejam disponíveis em hospitais públicos e privados, especulamos que o desconhecimento da evidência sobre o custo-efetividade das HBPMs como profilaxia de TEV leva a uma prescrição menos freqüente destas em hospitais públicos ${ }^{14}$. 
Tabela 3 - Fatores associados ao tipo de profilaxia farmacológica utilizada para tromboembolismo venoso

\begin{tabular}{|c|c|c|c|}
\hline Variáveis & $\begin{array}{c}\text { HNF } \\
\mathrm{N}=49(43,8 \%)\end{array}$ & $\begin{array}{c}\text { HBPM } \\
\mathrm{N}=63(56,3 \%)\end{array}$ & $\mathbf{P}$ \\
\hline Idade $\geq 40$ anos, $\%$ & 75,5 & 92,1 & 0,02 \\
\hline Gênero masculino, \% & 42,9 & 52,4 & 0,32 \\
\hline Raça, \% & & & $<0,0001$ \\
\hline Branca & 24,5 & 69,8 & \\
\hline Negra ou mulata & 75,5 & 30,1 & \\
\hline Tipo de hospital, $\%$ & & & $<0,000 \mid$ \\
\hline Público & 95,9 & 12,7 & \\
\hline Privado & 4,1 & 87,3 & \\
\hline Residência médica, \% & & & $<0,000 \mid$ \\
\hline Sim & 95,9 & 12,7 & \\
\hline Não & 4,1 & 87,3 & \\
\hline Contra-indicação para heparinas, \% & 46,9 & 14,3 & $<0,0001$ \\
\hline Diagnóstico principal é FR para TEV, \% & 59,2 & 77,8 & 0,03 \\
\hline$N^{0}$ de FR para TEV excluindo mobilidade reduzida, \% & & & 0,05 \\
\hline 0 & 10,2 & 9,5 & \\
\hline $1-3$ & 61,2 & 39,7 & \\
\hline$\geq 4$ & 28,6 & 50,8 & \\
\hline Mobilidade reduzida, $\%$ & 89,8 & 79,4 & 0,14 \\
\hline Tempo até início de profilaxia (dias), mediana (IQR) & $2(0-8)$ & $0(0-2)$ & 0,18 \\
\hline Duração de profilaxia & $16(9-43)$ & $9(3-27)$ & 0,05 \\
\hline \multicolumn{4}{|l|}{ Doses } \\
\hline \multicolumn{4}{|l|}{ HNF } \\
\hline $5.000 \mathrm{UI} I 2-12 \mathrm{~h}$ & $79,6(39)$ & - & \\
\hline $5.000 \mathrm{Ul} 8-8 \mathrm{~h}$ & $20,4(10)$ & - & \\
\hline \multicolumn{4}{|l|}{ HBPM } \\
\hline \multicolumn{4}{|l|}{ Enoxaparina } \\
\hline $20 \mathrm{mg}$ & - & $4,8(3)$ & \\
\hline $40 \mathrm{mg}$ & - & $79,4(50)$ & \\
\hline Outra $>40 \mathrm{mg}$ & - & $15,9(10)$ & \\
\hline Outras HBPM & - & 0 & \\
\hline Adequação de profilaxia & 20,4 & 95,2 & $<0,0001$ \\
\hline
\end{tabular}

A utilização de profilaxia para TEV em pacientes clínicos internados tem sido extremamente irregular também em hospitais e UTIs de estudos internacionais ${ }^{15,16,17,18}$. Recentemente, um estudo de coorte transversal em oito hospitais suíços mostrou que a utilização de profilaxia variava de $29 \%$ a $89 \%$, mas nenhuma diferença entre hospitais com ou sem residência foi demonstrada ${ }^{19}$. No presente estudo, o percentual de pacientes utilizando profilaxia foi semelhante entre hospitais públicos e com residência médica, e hospitais privados e sem residência médica. Entretanto, HNF foi prescrita mais comumente nos primeiros. Além disso, HNF foi usada mais freqüentemente em pacientes negros e mulatos, enquanto que HBPM para pacientes da raça branca. Atribuímos estes achados ao fato de os hospitais com residência médica, neste estudo, serem públicos, onde o percentual de pacientes negros e mulatos é maior. Entretanto, o uso de HNF foi significativamente menos adequado do que o de HBPM. Isso desperta a idéia de que hospitais públicos e com residência médica são alvos importantes para a disseminação de programas de educação sobre profilaxia de TEV em pacientes clínicos.
Este estudo faz parte de um esforço de especialistas em TEV e representantes de diversas sociedades médicas brasileiras para a compreensão da situação atual da profilaxia no Brasil. O projeto Endorse, um estudo multinacional de coorte transversal, está sendo desenvolvido em 2006, para estudar a utilização de profilaxia de TEV no Brasil e em outros 30 países. Após isso, planeja-se a disseminação de um programa educacional, em nível nacional, com recomendações sobre profilaxia, baseadas em evidência, a partir da Diretriz Brasileira para Profilaxia de TEV em Pacientes Clínicos. Em uma segunda fase, planejase uma reavaliação da situação das práticas de profilaxia de TEV nos mesmos hospitais recebendo programas educacionais baseados nesta diretriz, uma vez que o objetivo principal é aproximar a evidência científica sobre o uso de profilaxia da prática clínica.

\section{Conclusão}

FR para TEV são extremamente freqüentes em pacientes clínicos hospitalizados. Existe subutilização de profilaxia nesta população e também ampla variabilidade da profilaxia prescrita em hospitais públicos e privados. HBPM é utilizada mais adequadamente que HNF, entretanto, 
apenas a minoria dos pacientes clínicos hospitalizados e candidatos a profilaxia recebem prescrição adequada. Um programa educacional, em nível nacional, com recomendações sobre profilaxia a fim de aproximar a evidência da prática clínica é extremamente desejável.

Conflito de interesse: A primeira autora (ATCR) é consultora da Sanofi-Aventis.

\section{SUMMARY}

\section{INADEQUACY OF THROMBOPROPHYLAXIS IN HOSPITALIZED Medical Patients}

BACKGROUND. The risk of venous thromboembolism (VTE) is high in hospitalized patients, however itcan be reduced by adequate prophylaxis.

OBJECTIVE. To evaluate the adequacy ofVTE prophylaxis in hospitalized medical patients.

METHODS. A cross-sectional study was performed in hospitalized patients with acute medical illnesses in 4 hospitals of Salvador.

RESULTS. We evaluated 226 consecutive patients: 15.5\% in medical ICU, 79\% $\geq 40$ years of age and $48 \%$ male. The majority (97\%) had a least I risk factor (RF) for VTE, 79\% had reduced mobility and 62\% were diagnosed as having a RF at admission. Of the 208 prophylaxis candidates, $54 \%$ received some form of prophylaxis: unfractionated heparin (UFH) in $44 \%$, low molecular weight heparin (LMWH) in 56\% and mechanical methods in 2 patients. The utilization rate was similar in private and public hospitals. (51\% vs. 49\%), but LMWH was more common in private hospitals, withouta residence program (97\%), and UFH in the publicones (86\%). LMWH was more frequently used than UFH in patients $\geq 40$ years of age, more often in Caucasian than in Black patients, and less frequently in those with contraindications for heparin. Of the I 12 patients receiving prophylaxis, 63\% received adequate dosages: LMWH in $95.2 \%$ and UFH in 20.4\%. VTE prophylaxis was adequate in only $33.6 \%$ (70/208) of the patients.

CONCLUSION. Risk Factors for VTE were frequent in medical patients. There was considerable variability of the VTE prophylaxis prescribed in private and public hospitals. LMWH was used more appropriately than UFH. However, only a minority of patients candidates for prophylaxis, received adequate dosages. [Rev Assoc Med Bras 2006; 52(6): 44I-6]

KEY WORDS: Thromboembolism. Risk factors. Prevention and control. Internal Medicine. Heparin. Guidelines for medical practice.

\section{REFERÊNCIAS}

I. Kleber FX, Witt C, Vogel G, Koppenhagen K, Schomaker U, Flosbach $\mathrm{CW}$. Randomized comparison of enoxaparin with unfractionated heparin for the prevention of venous thromboembolism in medical patients with heart failure or severe respiratory disease. Am Heart J. 2003; | 45:6 | 4-2 I.

2. Kleber FX, Witt C, Flosbach CW. Study to compare the efficacy and safety of the LMWH enoxiparin and standard heparin in the prevention of thromboembolic events in medical patients with cardiopulmonary disease. Ann Hematol. 1998;76(Suppl):A93-5.
3. Samama MM, Cohen AT, Darmon JY, Desjardins L, Eldor A, Janbon C, et al. A comparison of enoxaparin with placebo for the prevention of venous thromboembolism in acutely ill medical patients. Prophylaxis in Medical Patients with Enoxaparin Study Group. N Engl J Med. 1999;34 I:793-800.

4. Geerts WH, Heit JA, Clagett GP, Pineo GF, Colwell CW, Anderson FA Jr, et al. Prevention of venous thromboembolism. Chest. 200I; I 19:132S-75S.

5. Alikhan R, Cohen AT, Combe S, Samama MM, Desjardins L, Eldor A, et al. Prevention of venous thromboembolism in medical patients with enoxaparin: a subgroup analysis of the MEDENOX study. Blood Coagul Fibrinolysis. 2003; | 4:34|-6.

6. Alikhan R, Cohen AT, Combe S, Samama MM, Desjardins L, Eldor A, Janbon C, Leizorovicz A, Olsson CG, Turpie AG. Risk factors for venous thromboembolism in hospitalized patients with acute medical illness: analysis of the MEDENOX Study. Arch Intern Med. 2004; I 64:963-8.

7. Risk of and prophylaxis for venous thromboembolism in hospital patients. Thromboembolic Risk Factors (THRIFT) Consensus Group. BMJ. 1992;305:567-74.

8. Geerts WH, Pineo GF, Heit JA, Bergqvist D, Lassen MR, Colwell CW, Ray JG. Prevention of venous thromboembolism: the Seventh ACCP Conference on Antithrombotic and Thrombolytic Therapy. Chest. 2004; 126:338S-400S.

9. Keane MG, Ingenito EP, Goldhaber SZ. Utilization of venous thromboembolism prophylaxis in the medical intensive care unit. Chest. 1994; 106:13-14

10. Goldhaber SZ, Dunn K, MacDougall RC. New onset of venous thromboembolism among hospitalized patients at Brigham and Women's Hospital is caused more often by prophylaxis failure than by withholding treatment. Chest. 2000; I 18: 1680-4.

II. Ryskamp RP, Trottier SJ. Utilization of venous thromboembolism prophylaxis in a medical-surgical ICU. Chest. 1998; I 13:162-4.

12. Arnold DM, Kahn SR, Shrier I. Missed opportunities for prevention of venous thromboembolism: an evaluation of the use of thromboprophylaxis guidelines. Chest. 200 |; | 20: | 964-7| .

13. Deheinzelin D, Braga AL, Martins LC, Martins MA, Hernandez A, Yoshida WB, et al. Incorrect use of thromboprophylaxis for venous thromboembolism in medical and surgical patients: results of a multicentric, observational and cross-sectional study in Brazil. J Thromb Haemost. 2006; 4: 1266-70.

14. Offord R, Lloyd AC, Anderson P, Bearne A. Economic evaluation of enoxaparin for the prevention of venous thromboembolism in acutely ill medical patients. Pharm World Sci. 2004;26:2। 4-20.

15. Aujesky D, Guignard E, Pannatier A, Cornuz J. Pharmacological thromboembolic prophylaxis in a medical ward: room for improvement. J Gen Intern Med. 2002; 17:788-9| .

16. Rashid ST, Thursz MR, Razvi NA, Voller R, Orchard T, Rashid ST, Shlebak AA. Venous thromboprophylaxis in UK medical inpatients. J R Soc Med. 2005;98:507-12.

17. Goldhaber SZ, Tapson VF. A prospective registry of 5,45 I patients with ultrasound-confirmed deep vein thrombosis. Am J Cardiol. 2004; $93: 259-62$.

18. Lacherade JC, Cook D, Heyland D, Chrusch C, Brochard L, Brun-Buisson C. Prevention of venous thromboembolism in critically ill medical patients: a Franco-Canadian cross-sectional study. J Crit Care. 2003; 1 8:228-37.

19. Chopard P, Dorffler-Melly J, Hess U, Wuillemin WA, Hayoz D, Gallino A, et al. Venous thromboembolism prophylaxis in acutely ill medical patients: definite need for improvement. J Intern Med. 2005;257:352-7. 